\title{
CONSIDERAÇÕES A RESPEITO DA DOENÇA DE PARKINSON DE INÍCIO PRECOCE
}

\author{
REVISÃO CRÍTICA DA LITERATURA
}

\author{
LUIZ AUGUSTO FRANCO DE ANDRADE*
}

\begin{abstract}
RESUMO - Desde sua descrição, em 1817, a doença de Parkinson sempre foi entendida como enfermidade que afeta predominantemente indivíduos acima dos 55 anos. Entretanto, desde o final do século passado descreveramse casos com início em idades precoces, abaixo dos 40 anos. Inúmeras séries de pacientes têm sido apresentadas, com as mais numerosas contando em tomo de 60 casos. Tem havido muita controversia a respeito de alguns aspectos. Um deles diz respeito à própria denominação, sendo chamado por uns como parkinsonismo de início precoce, parkinsonismo jovem por outros e, ainda, doença de Parkinson de ińcio precoce por outros tantos. Isto denota outra controvérsia sobre a verdadeira situação nosológica: trata-se da mesma doença de Parkinson, com as inclusōes dos corpos de Lewy, que ocorre nos indivíduos mais idosos ou, diferentemente, trata-se de outras entidades, com anatomopatologia diversa? Esta a razāo principal dos inúmeros estudos clínicos realizados nos pacientes deste grupo de início precoce, pois o número de estudos anatomopatológicos em tais pacientes é muito reduzido. Há evidências de que a maioria dos pacientes seja de doença de Parkinson clássica com início em idades mais jovens, apesar de uma série de descrições patológicas diferentes do habitual para esta enfermidade. Há maior encontro de casos familiares entre os pacientes jovens. Uma das características clínicas mais marcantes é o precoce e invariável aparecimento de complicações da levodopaterapia, como discinesias e flutuaçðes. Por outro lado, parece haver uma evolução clínica mais lenta e favorável. São apresentadas, revistas e comentadas grande parte das séries que apareceram na literatura.
\end{abstract}

PALAVRAS-CHAVE: doença de Parkinson, início precoce, parkinsonismo precoce, juvenil.

\section{A comprehensive critical review on early onset Parkinson's disease}

ABSTRACT - Since its original description Parkinson's disease has been considered as a clinical condition which affects older people. Nonetheless, since late in the last century, cases starting in very young age have been described. A great controversy has arisen concerning the real pathology in these cases and, consequently, how should they be named. Early or young onset parkinsonism, early or young onset Parkinson's disease, juvenile parkinsonism, all these terms have been used indistinguishable. There have been few pathological descriptions in early onset parkinsonism. Some show striking differences from the cases of older patients but others are very similar to what has been considered classical Parkison's disease. Younger starting age usually corresponds to greater possibility of other family members being affected. Dyskinesias and fluctuations due to chronic levodopa treatment are an early and almost invariable complication in the course of young patients. Comments on several aspects based on an extensive literature review are presented.

KEY WORDS: Parkinson's disease, young onset, juvenile, early onset parkinsonism.

*Professor Adjunto e Livre-Docente de Neurologia, Chefe da Disciplina de Neurologia da Escola Paulista de Medicina (Universidade Federal de São Paulo). Aceite: 6-agosto-1996. 
A doença de Parkinson (DP), desde que foi descrita por James Parkinson em $1817^{48}$, sempre foi entendida como enfermidade que afeta indivíduos na média-idade ou acima desta faixa etária. A maioria dos casos tem o início das manifestą̧ões clínicas nas $6^{\mathbf{4}}$ e $7^{\mathbf{2}}$ décadas de vida ${ }^{30}$. Um estudo cooperativo em cinco centros norteamericanos e um francês mostrou, num total de 1092 pacientes, média de idade ao início dos sintomas de 57,1 anos, variando de 53,4 anos em Toronto a 62,1 anos em Miami ${ }^{33}$. Curvas de incidência específica por idade de início mostram um pico entre os 60 e 70 anos, declinando progressivamente nos grupos mais idosos. Indivíduos muito idosos costumam desenvolver uma forma de parkinsonismo semelhante à $\mathrm{DP}$, que não responde à terapêutica com a levodopa, o que pode ser entendido como evidência de que se tratam de fenômenos de natureza diferente ${ }^{33}$. Casos de inicio mais precoce, entretanto, têm sido descritos em todas as grandes séries publicadas, com uma proporção que vai diminuindo progressivamente numa relação direta com a queda da idade de início.

O limite de idade a partir do qual se entende como doença de Parkinson habitual e abaixo do qual passase a denominá-la de DP de início precoce é arbitrário, a maioria dos autores admitindo como sendo 40 anos este limite ${ }^{15,22,23,49,55,67-70,24}$. Gershanik e Leist ${ }^{22}$.justificaram a escolha da idade de 40 anos como sendo equivalente a aproximadamente dois desvios padrōes abaixo da média de idade de todos os pacientes em sua série , a qual apresenta média de 61,3 anos e desvio padrāo de 11,8 anos.

Os primeiros casos de parkinsonismo de início precoce que apareceram na literatura foram os dois irmāos relatados por Siehr, em 1899 (apud Gershanik ${ }^{20)}$. Logo a seguir, em 1902, Bury relatou também dois casos familiares". Outros casos já haviam sido relatados anteriormente aos citados, mas houve controvérsias a respeito do diagnóstico, como o relatado por Huchard, em 1875 (apud Giovannini et al. ${ }^{2 n}$. Reunindo os casos apresentados desde 1850 e supostamente devidos a parkinsonismo de início muito precoce para a idade que se entendia como habitual para os casos de DP, acrescentando seus próprios casos, Willige, em 1911, coletou 46 casos de parkinsonismo, incluindo os casos de Siehr e de Bury, e selecionou 12 "ou possivelmente 14" casos que, em sua opinião se tratavam de DPts. Destes, 6 casos tinham familiares com DP. $O$ mais novo paciente tinha 18 anos de idade. Os casos familiares, em sua opinião, deveriam ser classificados num grupo separado da DP clássica e, inclusive, propôs para eles uma nova denominação, de "Paralysis Agitans Juvenilis Familialis".

Hunt $^{31}$, em 1917, descreveu quatro novos pacientes com quadro parkinsoniano com início precoce, nenhum deles com história familiar. A idade de início foi dos 13 aos 30 anos. Foi um trabalho extenso, com descrição clínica completa em um dos pacientes, que veio a falecer aos 40 anos, tendo iniciado sua enfermidade aos 15 . O curso da doença foi bastante característico, iniciando-se com tremor no membro superior e inferior esquerdos, ao longo do tempo acrescendo-se de rigidez muscular que se generalizou, com o tremor atingindo também o lado direito. Nas fases finais o paciente apresentava-se globalmente rígido e anártrico. Com sua morte, foi realizado exame necroscópico e o cérebro mostrou anormalidades apenas nos globos pálidos, onde havia perda neuronal. Devido a este achado solitário, Hunt chamou a doença de "atrofia primária do sistema palidal". Os outros três pacientes apresentavam quadros muito característicos de DP, um deles com hemiparkinsonismo.

Em 1923, Krabbe ${ }^{35}$ relatou uma série de pacientes com DP provenientes da Dinamarca, com 11 casos iniciando-se antes dos 40 anos, embora nenhum antes dos 30 anos. Ao lado disto, também nenhum apresentava história familiar positiva, da mesma maneira que os pacientes de Hunt. Alguns anos apos, um novo caso apresentando a atrofia progressiva do globo pálido, semelhante aquela descrita por Hunt, foi apresentado por Van Bogaert "4, num exame necroscópico de um paciente que havia iniciado tremor de tipo parkinsoniano aos 7 anos e que posteriormente desenvolveu outros sinais extrapiramidais. Entre estes, diferenciando-o muito de casos anteriormente descritos, havia manifestaçð̌es distônicas na forma de espasmos e movimentos corêicos.

Davison ${ }^{15}$, em 1947, descreveu 5 pacientes, sendo que um deles era uma mulher que fazia parte dos 4 casos originalmente descritos por Hunt em 1917. Os outros 4 eram membros de duas famílias e todos haviam iniciado seus sintomas abaixo dos 22 anos. $O$ caso não familiar que já houvera participado do trabalho de Hunt foi estudado anatomopatologicamente e Davison apresentou a todos eles como uma nova entidade que chamou de "doença pálido-piramidal", na qual os sintomas parkinsonianos precoces se seguiam de sinais piramidais, após longo tempo.

O trabalho que inaugurou a época moderna dos estudos epidemiológicos relacionados com a genética da doença de Parkinson, realizado por Mjones ${ }^{45}$ na Suécia, em 1949, identificou alguns casos de DP de início abaixo dos 20 anos (5 casos) e outros tantos abaixo dos 40 anos (e acima dos 20). Quatro dos pacientes com iń́cio abaixo dos 20 anos tinham outros familiares afetados. A técnica epidemiológica de identificar parentes 
com a mesma doença, baseada na existência de tremor apenas, tem sido muito criticada, pela possibilidade de se estar reconhecendo prováveis casos de tremor essencial como de DP. De outro lado, o estudo de Mjones, nāo apresentava descriçōes clínicas detalhadas, muito menos particularidades patologicas.

Outros casos familiares foram apresentados em 1958, quando Ota et al.47, relataram uma familia com três irmãos (2 irmăs e 1 irmão) que iniciaram um parkinsonismo juvenil aos 12, 13 e aos 20 anos. Uma das pacientes, faleceu aos 49 anos e seu cérebro foi estudado patologicamente. Os achados patologicos foram de anormalidades na substância negra, do tipo habitualmente encontrado nos casos de DP idiopática, além de perda neuronal no globo pálido, núcleo caudado, putamen e locus ceruleus. Os estudos patológicos até entāo mencionados costumavam encontrar anormalidades não típicas da DP idiopática clássica.

Casos ocasionais de pacientes muito jovens com DP juvenil continuaram a aparecer na literatura, em tempos mais recentes, como os dois irmãos descritos por Martin et al. ${ }^{43}$, em 1971. Estes pacientes iniciaram seus sintomas abaixo dos 20 anos ( 10 e 16 anos) e apresentavam quadro clínico característico da DP idiopática dos adultos, com rigidez muscular, bradicinesia, tremor de repouso e alterações posturais. $O$ caso 1 , que iniciou os sintomas aos 10 anos havia sido submetido a talamotomia esquerda aos 20 anos. Provavelmente foram os primeiros pacientes jovens a receber tratamento com a levodopa, que na época estava sendo introduzida no tratamento da DP. Houve excelente resposta terapêutica em ambos os irmãos, mas em questăo de poucos dias houve o aparecimento de movimentos involuntários anormais e flutuaçōes do rendimento terapêutico. Nesta época, as complicaçōes de longo curso do tratamento da DP com a levodopa ainda não eram bem conhecidas, muito menos os seus mecanismos fisiopatológicos. Nāo havia um padrão de resposta nos pacientes adultos que se pudesse

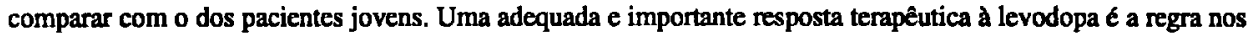
pacientes com a DP idiopática, que, inclusive, tem sido utilizada como um dos critérios de diagnóstico da enfermidade. Pacientes que respondem mal, ou simplesmente não apresentam resposta à terapêutica com a droga, embora possam ser casos atípicos da doença, são vistos como grandes candidatos a outras causas de parkinsonismo.

Houve séries em que os casos de início precoce eram isolados, sem antecedentes familiares, como na de Scott e Brody ${ }^{56}$, de 1971. Entre 21 casos de DP considerados por eles como de início precoce, 9 haviam iniciado antes dos 40 anos. $O$ curso clínico em todo o grupo era mais lento e benigno que em pacientes mais idosos. Houve uma consideração sobre a possibilidade de que, neste grupo, uma etiologia pós-encefalítica pudesse estar em jogo, a partir do fato de que a maioria dos pacientes houvera tido uma doença febril algum tempo antes do inf́cio dos sintomas, mas não havia sinais clínicos que justificassem esta possibilidade.

Uma outra peculiaridade encontrada por alguns autores em suas séries é a presença de flutuaçăo diuma dos sintomas, à semelhança com o fato já anteriormente descrito por Segawa e seus colaboradores ${ }^{51,5 \$}$. Estes autores relataram casos de pacientes jovens, crianças, com distonias geralmente se iniciando nos membros inferiores, que cursavam com marcada flutuação nos sintomas ao longo do dia, com caráter hereditário. Estes pacientes mostravam melhora espetacular com doses muito pequenas de levodopa. Em nosso meio, mais recentemente, foi publicado um caso de doença de Segawa ${ }^{3}$. A paciente iniciou os sintomas aos 18 anos, com uma forma espástica dos membros inferiores, que por anos foi diagnosticada como sendo uma forma de paraparesia espástica familiar de Strumpell-Lorrain (havia uma prima em segundo grau com este diagnóstico). Aos 28 anos, devido ao fato de haver flutuação diuma dos sintomas, foi sugerido o diagnóstico de doença de Segawa e um teste terapêutico com levodopa mostrou em três dias um efeito notável. Este grupo de pacientes foi o inicio do reconhecimento de todo um grupo mais amplo, hoje reconhecido, de pacientes com distonia-dopa-responsiva ("dopa-responsive-dystonia"), ou seja, que melhoram acentuadamente com a administraçāo da levodopa. Este grupo de pacientes, descrito em maior detalhe por Nygaard et al. ${ }^{46} \mathrm{em} 1988$, apresenta início com distonia, usualmente afetando a marcha, na infância ou adolescência, de mancira simultânea ou subsequente desenvolve sinais parkinsonianos e mostram dramática resposta à levodopa. Dados adicionais ao diagnóstico são a flutuação diurna, que nāo é encontrada em todos os pacientes, a melhora da distonia com o sono e a piora com o exercício e frequente história familiar sugerindo transmissāo autossômica dominante. Os quadros clássicos de distonia de torção não costumam responder à levodopa. Como alguns dos pacientes com distonia-dopa-responsiva nāo mostram flutuações diumas, isto motivou que a levodopa passasse obrigatoriamente a participar do esquema terapêutico inicial de qualquer caso de distonia idiopática, especialmente nas crianças. Com a distonia de torção num polo e a DP de início precoce no outro, há todo um espectro de apresentaçöes clínicas que se situam numa faixa intermediária entre os dois, podendo-se especular a relação patologica que possa haver entre eles, e o que leva a produzir estas formas transicionais entre a distonia idiopática e a DP. Quinze pacientes com parkinsonismo juvenil, cuja idade de início variou de 13 a 28 anos, foram relatados por Yamamura et al. ${ }^{65}$, 
sendo que 10 deles apresentavam flutuaçōes diumas nos sintomas parkinsonianos. Todos estes 10 pacientes eram do sexo ferninino, que, aliás, predominava no grupo ( 13 do sexo feminino e 2 do sexo masculino). Todos os pacientes pertenciam a quatro famílias. Outro fato muito frequente, foi que nas pacientes que apresentavam flutuaçð̃es diumas, havia franca melhora após período breve de sono, mesmo por 30 minutos. Além do aspecto genético envolvido nestes pacientes, havia também fenômenos distônicos nos pés (pés equinos). Era a época inicial do uso da levodopa e apenas um dos pacientes havia usado a levodopa, com resultados espetaculares.

A resposta espetacular ao tratamento com a levodopa, assim como o desenvolvimento precoce de discinesias da levodopa, também foi apontada por Sachdev et al. ${ }^{53} \mathrm{em}$ seus três casos. Havia os casos de duas irmãs que iniciaram os sintomas aos 10 e 9,5 anos c um terceiro caso, iniciado aos 5 anos, em consequência a encefalite ocorrida durante uma infecção, a qual foi diagnosticada como sendo varicela, mas que pela descriçăo nos pareceu outra doença viral aguda, exantemática, de outro tipo que não a varicela, pela caracteristica da erupção cutânea. Os 4 casos familiares descritos por Carlier e Dubru' ${ }^{13}$, em 1979, com início bastante precoce (aos 14 anos), da mesma maneira, mostraram melhora muito pronunciada com a levodopa e, igualmente, desenvolveram precocemente quadro discinético relacionado à droga.

Em 1979, Yokochi ${ }^{67,68}$ iniciou uma série de artigos a respeito de DP de início precoce, que foram sendo publicados nos anos seguintes, sugerindo a denominação de parkinsonismo juvenil para os casos de DP idiopática com início abaixo dos 40 anos. A idéia de Yokochi, desde o início, era de que havia importantes diferenças clínicas, de resposta terapêutica e possivelmente também neuropatológicas entre os casos de parkinsonismo juvenil, como ele e subsequentemente outros também chamavam os casos de DP de início precoce. Com as características clínicas e de resposta terapêutica que Yokochi e Narabayashi ${ }^{69}$ analisaram em 1981, foram capazes de sugerir uma classificação, baseada nestes dois aspectos, dos pacientes com DP de início precoce, em três tipos básicos: tipo l, pacientes com apresentaçāo clínica e curso similar ao que ocorre nos casos de DP idiopática de início mais tardio, como habitual, porém com uma resposta terapêutica à levodopa mais dramática; tipo 2 , neste, os pacientes são similares aos do tipo 1 no que concerne à idade e sintomas, mas a resposta à levodopa é menos evidente; tipo 3, aqui se concentraram casos de início muito mais precoce (abaixo dos 20 anos, entre os 6 e os 16 anos), cujos sintomas habitualmente se iniciavam nos membros inferiores, com manifestaçōes distônicas e posturas anormais, como pé equino. Estes pacientes mostraram as mais dramáticas respostas favoráveis à levodopa. Yokochi e Narabayashi preocuparam-se em pesquisar os pacientes do ponto de vista patológico, para determinar se se tratava de enfermidade com o mesmo substrato patologico que a DP idiopática dos pacientes mais idosos ou, na verdade, tratava-se de alguma outra patologia, como sugeriam os trabalhos anteriores de Hun $^{31}$ e Van Bogaert ${ }^{64}$. Outro aspecto que eles captaram em sua série 6 a diferença de prevalência dos casos precoces no grupo total de pacientes com DP, notada entre os casos do mundo ocidental e os do Japão, maior neste. As séries japonesas acusam entre $10 \mathrm{e} \mathrm{18,4 \% 69}$, enquanto as ocidentais costumam variar entre 5 e $10 \% 23,00,63$.

A contribuição brasileira pioneira em publicaçāo específica no tema, de nosso conhecimento, além da de Araújo \& Miranda ${ }^{3}$ que relataram caso de doença de Segawa, foi trazida por Scaff et al.s, em 1980. Estes autores relataram 10 pacientes com parkinsonismo juvenil, adotando como critério de inclusăo pacientes abaixo de 30 anos de idade quando do início dos sintomas, com idades limites de 9 e 29 anos. Eram 7 do sexo masculino e 3 do feminino. Três casos tinham parentes com DP ( o caso 2 tinha uma irma com uma síndrome parkinsoniana e os casos 9 e 10 cram irmāos, com referência a um terceiro irmão com doença semelhante). Os demais casos nāo apresentavam parentes com DP. Apenas um caso não apresentava tremor, 8 exibiam a tríade clássica e um não tinha rigidez. De 9 casos tratados com levoropa, 8 tiveram "nítida melhora do quadro". Apenas um paciente apresentou quadro sugestivo de distonia nu membro superior como manifestaçāo inicial (caso 2). Nāo hă inferência sobre a prevalência destes casos no total ve seus pacientes parkinsonianos.

Casos isolados de DP de início precoce também apareceram na literatura, como o descrito por Clough et al. ${ }^{14}$,em1981. Este caso, uma paciente que começou a apresentar sintomas parkinsonianos aos 15 anos, desenvolveu todas as características de doença de Parkinson idiopática. Não tinha história familiar positiva e nunca desenvolveu sinais neurológicos at́picos. Foi investigada amplamente e apenas se encontrou diminuição da quantidade de HVA (ácido homovanílico) no líquor, na faixa do habitual para a DP idiopática. A resposta ao tratamento com a levodopa foi muito boa e apés algum tempo apareceram discinesias e deterioração de fim de dose.

No Canadá, em 1982, Barbeau e Pourcher ${ }^{7}$ realizaram estudo epidemiológico e genético dos seus casos de início precoce, apresentando 35 pacientes com idade de início abaixo dos 40 anos. Eles indicavam prevalência de $9,4 \%$ no total dos z:us casos, porém, como Gershanik ${ }^{20}$ aponta, esta taxa devia refletir uma prevalência sobre apenas uma parte do total de pacientes (342 pacientes), os quais vinham efetivamente sendo seguidos e 
não sobre o total de 713 conforme apontam os autores como sendo o número total do seu arquivo. Se os 35 pacientes com início precoce foram coletados em todo o material, a taxa, recalculada deve ser de 4,9\% (e não de 4,5\% como diz Gershanik). Uma diferença importante desta série em relação às demais é o fato de que $23 \%$ dos pacientes não terem apresentado qualquer resposta ao tratamento com a levodopa. Apresentam $32 \%$ de casos familiares, porem incluindo 8 casos em que os familiares tinham tremor familiar (tremor essencial?).

Outra contribuiçāo de autores de língua portuguesa à literatura foi a trazida por Lima et al. ${ }^{41}$, de Portugal, que apresentaram uma série de 21 pacientes em simpósio, realizado em New York (1985) e posteriormente a publicaram ${ }^{42}$. Os 21 casos representavam 9,2\% do total de seus casos de DP. História familiar positiva para DP foi encontrada em apenas dois pacientes (aproximadamente 10\%) e em outros três, havia história de tremor familiar (tremor essencial?). Seus pacientes tiveram boa resposta ao tratamento com levodopa. Apenas 6 pacientes atingiram o estágio IV e V da escala de Hoehn e Yahr.

Gershanik e Leist ${ }^{22}$, conforme já citado anteriormente, utilizaram o artifício de limitar a idade de inf́cio a 40 anos, como sendo duas vezes o desvio padrăo para baixo da idade média do seu total de pacientes parkinsonianos (média 61,3 anos; desvio padrão de 11,8 anos), porém reduziram mais 5 anos, como medida de segurança, atingindo entāo a idade limite de 35 anos para seus casos. Com esta limitaçăo, chegaram a 13 pacientes com "DP de início juvenil" ("juvenile onset Parkinson's disease"), a denominação que usaram nesta primeira publicação. A partir de 1988, Gershanik e seu grupo preferiram passar a utilizar a denominaçăo de "parkinsonismo de inicio precoce" ("parkinsonism of early onset"), pelas dúvidas sobre se os casos realmente se devem à doença de Parkinson, ou a outras entidades nosológicas, julgando-se pelas diferenças patológicas apresentadas nos poucos relatos de estudos patológicos, além das diferenças clínicas e genéticas tambem vistas nos diversos trabalhos já apresentados ${ }^{20}$. Esta série representava $3,8 \%$ do total de seus casos de DP. Os pacientes melhoraram significativamente com o uso de levodopa e mostraram uma tendência precoce de desenvolver complicaçōes do tratamento com a droga. Um detalhe importante desta série foi a proporção elevada de pacientes com manifestações distônicas (53\%), algumas antecedendo os sintomas parkinsonianos. Não ficou claro no trabalho se estas manifestaçð̃es eram definidamente nāo relacionadas à terapêutica com a levodopa. $A$ incidência de antecedentes familiares com DP era baixa, com apenas três casos, o que não diferia do que se admite ser esperado para casos de DP de início mais tardio.

A maior série até a época, com 60 pacientes, foi a de Quinn et al. ${ }^{49}$, em 1987. Os autores fizeram uma separação de seus casos entre os que iniciaram os sintomas com menos de 21 anos, aos quais denominaram de parkinsonismo juvenil ("juvenile parkinsonism") e os que iniciaram entre os 21 e 39 anos, aos quais denominaram de DP de início jovem ou precoce ("young-onset Parkinson's disease"). Note-se que os autores introduziram modificação no conceito etário do que se denominava parkinsonismo juvenil, trazendo este rótulo somente para os casos de infcio muito precoce. De qualquer maneira, neste grupo havia apenas 4 pacientes. Nos pacientes do grupo de idade de início entre os 21 e 39 anos não foi encontrada incidência familiar maior que a esperada para casos de idade superior. Dentre 4 casos "juvenis", em três havia algum irmão acometido pela mesma enfermidade. Houve acentuada resposta favorável com o uso da levodopa em todos os pacientes, mas após 6 anos de evoluçăo, $100 \%$ exibiam discinesias e/ou flutuações. Não houve estudo patológico em nenhum paciente, mas os autores especulam que os casos de início muito precoce (abaixo de 21 anos) devem ser considerados como sendo outra enfermidade que não a DP, talvez relacionados com os casos de parkinsonismo-distonia (distonia-dopa-responsiva de Nygaard et al.6. distonia familiar com flutuaçoes diumas de Segawa et al. ${ }^{57.34}$ ). Os autores estavam, desta maneira, interpretando estes casos como não pertencendo a um subgrupo extremamente jovem de DP, mas sim a alguma outra enfermidade.

Durante o XIII • Congresso Brasileiro đe Neurologia, realizado em 1988 em São Paulo, Sardinha et al."* fizeram uma comunicaçăo, relatando três casos de parkinsonismo juvenil familiar, irmãos entre si, com início dos sintomas antes dos 19 anos. Segundo os autores, "observando-se na fase inicial um predomínio de tremor, aparecendo posteriormente bradicinesia e rigidez, sem outros sinais ou sintomas neurológicos". Não encontramos a publicação destes três casos.

Num trabalho detalhado, fazendo comparaçðes clínicas e patol6gicas entre um grupo de pacientes de DP com início precoce e outro com início bastante tardio, Gibb e Lees ${ }^{24}$, em 1988, trouxeram importante contribuição ao assunto. $O$ critério idade de ińcio para o grupo jovem foi um pouco menos restritivo, pois incluiram pacientes de até 45 anos. $O$ dos idosos incluiu pacientes de 70 anos para cima. No grupo jovem havia 46 pacientes e no idoso, 52. A média de idade de iń́cio no grupo jovem foi 38 anos, variando de 24 a 45 anos. No grupo idoso, a média foi 73 anos, variando de 70 a 90 anos. Apenas três pacientes do grupo jovem tinham 
parentes de $1^{\circ}$ grau com DP e foi considerado como não apresentando diferenças com o grupo idoso, mas devese considerar que a idade mais baixa dos pacientes jovens foi 24 anos. Uma elevada frequência de discinesias em ambos os grupos foi observada, quando se comparava levando-se em consideração pacientes tomando doses similares de levodopa, sendo $91 \%$ nos jovens e $69 \%$ nos idosos. Dos pacientes avaliados clinicamente, um terço no grupo de jovens desenvolveu distonias, principalmente nos pés. Nenhum paciente do grupo dos idosos (em 13 avaliados) desenvolveu a complicação. Do ponto de vista clínico os autores chegaram à conclusāo de que os dois grupos apresentavam diferenças apenas na maior frequência de dificuldade à marcha como sintoma inicial nos idosos, e sensação de rigidez muscular, distonia nos períodos "off" (quando desaparece o efeito da levodopa) e duração mais longa da enfermidade, os três predominando no grupo mais jovem. Outra contribuição importante dos autores foi o estudo patologico, realizado em dois grupos de cérebros, pertencentes a outros pacientes, já que nenhum dos incluidos no estudo clínico morreu durante a realização. Estudaram 12 cérebros de pacientes que haviam tido DP de início precoce, cujas idades variaram de 23 a 40 (média de 34 ao iń́cio) e 22 cérebros de pacientes que haviam tido DP de início senil, cujas idades variaram de 70 a 87 anos (média de 76 ao início). Embora os detalhes dos achados patológicos só iremos reportar posteriormente, podemos adiantar que os autores apenas encontraram $24 \%$ maior perda de neurônios da substância negra no grupo dos jovens, com praticamente a mesma patologia básica dos corpúsculos de Lewy em ambos os grupos, levando-os à conclusão final de que tanto clínica como patologicamente os dois grupos se comportaram de maneira similar, devendo ser encarados como devidos à mesma doença.

Em 1990, Askenazy et al. ${ }^{4}$ relataram 4 casos de Israel, todos abaixo de 30 anos, que apresentaram parkinsonismo juvenil com distonia. Os dois primeiros casos eram irmãos; os demais eram isolados. No caso 3 , os pais eram consanguíneos. Os dois irmãos e o terceiro caso, iniciaram os sintomas com distonia nos membros inferiores, aos 13, 13 e 12 anos respectivamente, sendo que o quarto caso apresentou distonia do pé após alguns anos do início, que se deu aos 25 anos. Não havia menção a flutuaçóes diurnas nos sintomas. Os 4 revelaram marcada resposta à terapêutica com a levodopa, sendo que em três deles houve desenvolvimento de discinesias e flutuaçōes do rendimento terapêutico após menos de 1,3 e 4 anos, respectivamente. Os autores concluem que a marcada responsividade à levodopa em doses reduzidas é o maior marcador da DP juvenil.

Em 1991, Kostic et al. ${ }^{34}$, em Belgrado, estudaram as discinesias e as flutuações do rendimento terapêutico nos pacientes em uso de levodopa buscando diferenças entre pacientes com início precoce ou com início na faixa etária habitual. Utilizaram o mesmo critério de idade de início para o grupo mais jovem que o utilizado por Quinn et al., considerando como doença de Parkinson de início precoce pacientes entre 21 e 40 anos. Este grupo, consistia de 25 pacientes, embora o registro total de pacientes habilitados a entrar no estudo fosse de 44 . não tendo sido utilizados no seu total por não se ter conseguido pareá-los com casos de idade superior em número suficiente. A média de idade foi 33,5 anos (desvio padrão de 5,5 anos), mas as idades extremas não foram informadas. No grupo de início mais idoso, entraram 25 pacientes com idade de início acima de 40 anos. A média de idade foi 55,8 anos (desvio padrão 5,2 anos) e também não informaram as idades limites. Em cada grupo havia proporçāo feminino/masculino de 14/11, com predominância do sexo feminino, como no grupo de Yamamura et al. ${ }^{\mathrm{s}}$. Os pacientes de ambos os grupos foram perfeitamente pareados na duraçăo da doença $(9,1$ anos), na frequência do sintoma inicial, na graduaçăo da escala de Hoehn e Yahr (2,96 e 2,98), na duração do tratamento com a levodopa (7,28 anos) e na dose da droga (608 e $605 \mathrm{mg} / \mathrm{dia})$. 0 trabalho confirmou que os pacientes de início precoce têm mais discinesias e flutuaçōes em consequência ao tratamento com a levodopa que os de início mais tardio, assim como as complicaçð̃es se apresentaram mais precocemente no grupo de idade mais baixa, recomendando que se retarde o máximo que se possa a introdução da levodopa no regime terapêutico dos pacientes jovens.

Ainda em 1991, outro estudo comparativo entre grupos de pacientes jovens e com idade mais elevada foi publicado ${ }^{27}$. A motivação do trabalho foi a mesma que mobilizou outros pesquisadores recentes, com a tentativa de confrontar os aspectos clínicos de pacientes jovens e de idade mais avançada e poder definir os pontos chaves que os diferenciem. Os autores, Giovannini et al., de Milão, estudaram três grupos de pacientes parkinsonianos, todos com diagnóstico clínico de DP idiopática. Num primeiro grupo, alocaram 60 pacientes, aqui também utilizando critérios etários de inclusão semelhantes aos de Quinn et al. ${ }^{49}$, com idade de início entre os 20 e os 40 anos; no segundo grupo, foram incluidos 60 pacientes com idade de início acima dos 40 anos. Estes dois grupos foram comparados segundo diversas caracteristicas clínicas, de evolução e de resposta terapêtica. Para observar diferenças de prevalência de demência ou efeitos colaterais psiquiátricos do tratamento com a levodopa, os pacientes do primeiro grupo foram comparados com um terceiro grupo de 134 pacientes consecutivos, não selecionados, com idades de 45 a 80 anos, ao início. Os pacientes de inf́cio precoce 
representavam $10,2 \%$ do total de pacientes com DP. Apenas três pacientes neste grupo tinham parentes de $1^{\circ}$ ou $2^{\circ}$ graus com DP (5\%). Havia 38 homens e 22 mulheres nos dois primeiros grupos e 73 homens e 61 mulheres no terceiro grupo. Algumas características clínicas algo diversas das encontradas em outras séries foram observadas. Alteraçðes autonômicas, pouco encontradas por outros autores, aqui predominaram no grupo dos jovens $(83,3 \%)$, em relação ao segundo grupo $(58,3 \%)$. Uma progressão "lenta" da enfermidade foi característica do grupo mais idoso e uma evolução mais "rápida" caracterizou o grupo mais jovem. $\mathbf{O}$ grupo mais idoso teve melhor e mais duradoura resposta medicamentosa à levodopa que o grupo jovem, e as complicações motoras de longo prazo foram mais comuns no grupo jovem (78\%) que no idoso (62\%), embora esta diferença não atingisse significância estatística. Do ponto de vista de manifestaçס̄es psíquicas e demência, o terceiro grupo foi significantemente mais afetado que os jovens. Foram avaliados também alguns "fatores de risco" (fumar cigarros, antecedentes de vida rural até os primeiros 15 anos de idade e profissão), que não se mostraram relevantes. Chegaram à conclusão de que os pacientes de início precoce não apresentaram diferenças fundamentais em relação aos pacientes de início mais tardio, apenas com maior tendência de evoluir mais rapidamente para um quadro completo de parkinsonismo.

As controvérsias que se tem provocado ao se considerar o grupo dos pacientes jovens com parkinsonismo ou DP como tendo diferenças dos pacientes de grupo etário superior, ainda têm motivado os autores a estudar melhor os mais jovens. Com este intuito em mente, o grupo português, liderado por Antonio Bastos Lima (Porto), publicou pesquisa enfocando apenas os pacientes com DP de início até os 30 anos ${ }^{\mathbf{4}}$, a exemplo do que fizera o primeiro trabalho de revisão da literatura ${ }^{65}$. Os 19 pacientes estudados representavam $31,1 \%$ do total de pacientes abaixo de 40 anos, coletados em duas instituiçōes neurológicas portuguesas. Havia 10 pacientes do sexo masculino e 9 do sexo oposto. A média de idade de iń́cio era 24,7 anos, variando de 12 a 29 anos (abaixo de 20 anos, apenas dois casos). Apenas um paciente, o que teve o início mais precoce, aos 12 anos, apresentouse com sintoma inicial de distonia no membro inferior. Não havia distúrbios autonômicos no grupo. História familiar positiva para DP foi constatada em apenas dois pacientes, assim como de tremor essencial em outros dois. De 15 pacientes que persistiram no tratamento com a levodopa, 10 desenvolveram complicações motoras do tratamento, além de dois que pararam a medicaçāo precisamente por este motivo.Os autores sustentam a idéia, obtida a partir deste estudo, de que os pacientes mais jovens, abaixo de $\mathbf{3 0}$ anos, têm curso mais lento da doença com boa e mantida resposta ao tratamento com a levodopa, como muitos dos trabalhos anteriores ja haviam citado, porém diferente, por exemplo, do de Giovannini et al. ${ }^{27}$, anteriormente citado. Outro fato diferente do apontado em muitas outras séries foi o tempo de latência para o início das complicações motoras da levodopa mais prolongado nesta série $(5,5$ anos $)$.

Ainda nesta linha de tentar demonstrar uma diferença entre parkinsonianos jovens e mais iđosos, o que tem sido tentado por comparaçōes clínicas ou patológicas, nos raros casos de pacientes submetidos a exame necroscópico, o grupo liderado por Narabayashi e Yokochi, de Tokyo, utilizando o líquor ventricular retirado durante ventriculografias previas a cirurgias estereotáxicas para o tratamento da DP, estudaram o conteúdo de biopterin total neste líquido ${ }^{19}$, comparando-o com o de pacientes controle. $O$ biopterin total espelha o conteúdo cerebral de (6R)-L-eritro-5,6,7,8-tetra-hidrobiopterin (BH4), o qual e o cofator natural para a tirosina-hidroxilase e a triptofano-hidroxilase. O BH4 é altamente concentrado nos terminais dopaminérgicos nigroestriatais e a diminuição da concentração de biopterin total no líquor ventricular de pacientes com a forma clássica de DP, se supōe, reflete o grau de degeneração dos neurônios nigroestriatiais dopaminérgicos ${ }^{19}$. Foram estudados 19 pacientes com DP com início dos sintomas antes dos 40 anos (grupo jovem) e 61 pacientes com a DP clássica, com início acima desta idade. Os pacientes jovens, ainda, de acordo com a resposta terapêtica à levodopa, foram divididos em dois subgrupos: I, com boa resposta terapêutica seguidos por marcadas flutuaçðes e discinesias, como tem sido apontado para os pacientes mais jovens; II, com resposta terapêutica menor e menos flutuaçðes e discinesias, sendo mais similar a DP clássica. O conteúdo de biopterin total ventricular, tanto no grupo de parkinsonianos jovens como no dos mais idosos estava significativamente diminuido em relaçăo aos controles. Entretanto, estava marcadamente mais diminuido nos pacientes do subgrupo jovem I. Isto foi interpretado como uma possibilidade de que os pacientes do subgrupo jovem II (os mais semelhantes à DP clássica na sua reaçāo à levodopa) fossem apenas um prolongamento, em faixas etárias inferiores, da patologia clássica da DP e os pacientes jovens do subgrupo I pudessem ter uma patofisiologia diferente da DP clássica, com maior comprometimento da capacidade de sintetizar dopamina.

Uma série de evidências, às quais vamos adiante nos reportar, vem apontando para a possibilidade de que fatores ambientais, como determinadas atividades profissionais, exposiçāo crônica a dețerminados contaminantes ambientais, antecedentes de vida rural prolongada nos primeiros anos da vida, ingestāo de água 
de poço, contacto com agrotóxicos (pesticidas) e outros fatores do gênero, possam estar relacionados de alguma forma à patogênese da doença de Parkinson, especialmente nos pacientes mais jovens. Tentando encontrar indícios que pudessem trazer mais fundamento a esta hipótese de patogênese da DP, um grupo de Portland (Oregon, USA) publicou estudo relacionando vários destes fatores ambientais com um grupo de 63 pacientes de DP com início em idade jovem ("young-onset Parkinson's disease"). Utilizaram outro critério etário que os encontrados até entāo na literatura, incluindo pacientes que tiveram seu diagnóstico (e não a idade de início) de DP até a idade de 50 anos, inclusive, admitindo que todos são representativos de um início mais jovem que o habitual' ${ }^{2}$. Certamente com isto, por ser menos restritivo o critério de inclusão, aumentaram muito sua amostra. Năo há detalhes clínicos, tratando-se de estudo epidemiológico em que os diagnósticos foram confirmados por telefone com os próprios pacientes ou através de carta com o seu neurologista responsável. Este grupo de pacientes foi confrontado com outro, controle, consistindo de 68 pacientes com artrite reumatóide, pareados por idade, sexo, ano de nascimento $e$ ano de diagnóstico. Ambos os grupos são formados pelos pacientes que responderam a volumoso questionário de exposição ambiental, que havia sido enviado a um contingente de 100 pacientes com DP e $186 \mathrm{com}$ artrite reumatóide. Vários itens estudados mostraram resultados significantes do ponto de vista estatístico. Dezesseis pacientes com DP $(25,4 \%)$ tinham história familiar para DP, sendo que 7 controles $(10,3 \%)$ tambem tinham este item positivo, sendo esta diferença significativa. Outros dados positivos foram: exposição a pesticidas, antecedente de ter vivido em casa fumigada com tóxicos, exposição a herbicidas, vida rural na época do diagnóstico e ter antecedente de se alimentar com sementes ou caroços ("nuts") desde 10 anos antes do diagnóstico. Uma relação inversa, significante, foi verificada em relação a ser furnante, por vários períodos de tempo antes do diagnóstico, vida rural prévia e exposição ao dimetil sulfóxido. Para os autores, seus dados eram consistentes com a hipótese ligando a gênese da DP com fatores de exposiçăo a agentes pesticidas ou relacionados a eles.

Vamos passar agora a nos reportar aos estudos patológicos que têm sido realizados em pacientes com parkinsonismo ou com DP de início precoce, que foram realizados na tentativa de esclarecer se eles se tratam de DP iniciando-se em idades jovens ou se se tratam de casos consignáveis a outras entidades nosológicas. Houve número relativamente pequeno dẹ estudos patológicos em pacientes deste grupo ao longo dos anos, portanto talvez não sendo significativo para exprimir todo o conjunto de casos. Como já apontamos no início deste trabalho, o primeiro caso analisado patologicamente foi o de Hunt" em 1917, denominado por ele de "atrofia primária do sistema palidal", o qual revelou atrofia difusa, com perda de células nos globos pálidos, além de perda de células no neoestriado, com algum envolvimento do núcleo basal de Meynert, aparentemente sem comprometimento da substância negra ${ }^{31}$. O caso estudado por Van Bogaert ${ }^{\text {t4 }}$, em 1930, era semelhante ao de Hunt $^{31}$, porém com redução do número de células e atrofia celular na substância negra, menos importante do que o usual nos casos de DP senil ${ }^{\text {s4 }}$. Em 1947, Davison ${ }^{15}$ realizou autópsia num dos 4 pacientes originais de Hunt, urna senhora que ao longo dos anos, apresentou também sinais piramidais ao lado dos parkinsonianos. Além das alteraçōes palidais anteriormente descritas, Davison encontrou alterações na substância negra, além de desmielinização dos feixes corticoespinhais, o que o levou a denominar seus casos (havia mais 4 com sinais piramidais que não foram estudados patologicamente) de "doença pálido-piramidal". Em 1958, Ota et al."7, apresentaram a primeira descrição de estudo patológico com alterações semelhantes às habitualmente vistas nos casos clássicos de DP, com despigmentação da substância negra, perda neuronal em vários núcleos da base, locus ceruleus e também perda neuronal na camada granular do cerebelo. Não fizeram menção ao encontro de corpúsculos de Lewy na substância negra. $O$ cérebro pertencia a um paciente com evolução clínica de mais de 30 anos. Isto deve ser levado em conta ao se fazer análises comparativas em patologia de doenças degenerativas ou sujeitas a ação crônica de contaminantes ambientais. Outro fato que deve ser relevante é quanto o próprio aumento de idade e os fenômenos de envelhecimento podem interferir na patologia básica e na sua expressividade histopatológica.

Os trabalhos de Yokochi, Narabayashi e seus colaboradores, da Juntendo University School of Medicine. de Tokyo, já anunciados anteriormente, vieram trazer grande contribuiçāo ao estudo clínico, farmacológico, bioquímico, cirúrgico e patológico da DP de início precoce, que eles chamaram de parkinsonismo juvenil. Em 1984, publicaram os resultados clínicos e farmacológicos de um grupo de 40 pacientes, que, como vimos anteriormente, eram divididos em três categorias; numa primeira, com casos semelhantes aos da DP clássica, porém com início mais precoce e resposta mais dramática à levodopa; numa segunda, com casos similares aos da primeira, porém com menor resposta à levodopa; no terceiro, casos mais jovens ainda, que se iniciam com distonia nos membros inferiores ${ }^{\circ}$. Apresentaram os achados anatomopatológicos de dois casos, um do segundo e um do terceiro grupo da sua classificação. No caso do segundo grupo, encontraram perda dos neurônios pigmentados da zona compacta da substância negra e do locus ceruleus, com inclusões de corpúsculos de Lewy, 
indistinguiveis do que se encontra nos casos clássicos de DP. Entretanto, no segundo caso estudado, pertencente a paciente do terceiro grupo, com distonias e início mais precoce, os achados neuropatológicos foram muito distintos. Macroscopicamente, havia grande redução da pigmentação da substância negra, porém, microscopicamente, foi encontrada uma população de células nigrais praticamente normal em quantidade, porém. com intensa diminuição de pigmentação, com células arredondadas, parecidas às encontradas em crianças normais, antes do início da pigmentação melânica natural. A paciente, entretanto já contava 39 anos. Havia poucas inclusões de corpúsculos de Lewy. $O$ locus ceruleus apresentava alteraçð̃es semelhantes às da substância negra e não havia anormalidades em outras estruturas. Os estudos bioquímicos que haviam realizado mostravam que este segundo caso apresentava, destarte, intensa diminuição de atividade da tirosina-hidroxilase no caudado, putamen e locus ceruleus, embora fosse quase normal na substância negra. Os autores concluiram que as alteraçzes morfológicas e bioquímicas sugeriam que os dois casos representavam duas diferentes entidades dentro da moldura do parkinsonismo juvenil. Alguns anos depois o material do segundo caso, da paciente do terceiro grupo, foi reestudado, com a ajuda de Gibb e Lees, de Londres, à luz de conhecimentos atuais sobre as características das lesð̄es nigrais na DP. Este grupo demonstrou que na DP existe um dano preferencial nos neurônios na região ventrolateral da substância negra, pars compacta, a qual é pobremente melanizada, exibindo maior vulnerabilidade ${ }^{26}$. Os resultados deste reexame do caso de Yokochi et al.$^{70}$ foram apresentados durante 0 Meeting da American Academy of Neurology de $1989^{25}$ e posteriormente publicados ${ }^{26}$. Os novos resultados apontavam para destruição total da porção ventrolateral da substância negra (pars compacta), com número relativamente normal de células nigrais em outras partes, a despeito de alguns corpúsculos de inclusão de Lewy. O conteúdo de melanina nas células remanescentes correspondia ao nível normal para o intervalo de idade entre os 5 e 10 anos. A paciente ao falecer contava 39 anos. O locus ceruleus mostrava poucos corpúsculos de Lewy mas sem perda de células e os demais sítios cerebrais estudados estavam livres de anormalidades. Concluiram que o padrão aqui apresentado, de perda seletiva de neurônios na porção ventrolateral da substância negra é idêntico, embora mais pronunciado, ao encontrado nos casos clássicos de DP do adulto de idade mais avançada. A presença dos corpúsculos de Lewy e a vulnerabilidade seletiva dos neurônios nigrais demonstrada implica que as entidades parkinsonismo juvenil com distonia e a DP têm mecanismos patogenéticos semelhantes. Se se tratam da mesma doença, manifestando-se apenas em grupos etários diferentes, com expressividade diferente devido à imaturidade celular, ou, ainda, se o efeito do envelhecimento celular modifica o padrão de alteração produzido pelo processo patológico básico, são pontos ainda a esclarecer. Se o defeito genético básico envolvido nos pacientes parkinsonianos de início precoce, com história familiar positiva, for identificado, isto poderá muito contribuir para que a causa da DP seja elucidada.

Dois casos algo diferentes de parkinsonismo juvenil familiar foram apresentados em 1986 por Mayer et al. ${ }^{44}$, num estudo clínico-patológico. Os autores relataram dois irmãos, que aos 19 e 24 anos de idade iniciaram sintomas parkinsonianos, o primeiro com forma rígido-acinética, apenas corn certo tremor postural e o segundo, com tremor de repouso. Ambos tiveram evolução muito arrastada ao longo de muitos anos. $O$ primeiro, aos 48 anos apresentava, além do quadro parkinsoniano, arreflexia tendinosa global nos 4 membros, com sensibilidades vibratória e cinético-postural muito diminuidas abaixo dos joelhos e sinal de Romberg. Um eletrorretinograma mostrou degeneração retiniana importante. A levodopa mostrou resposta dramática, sem flutuaçð̃es, mas aos 56 anos o paciente faleceu devido a adenocarcinoma de pâncreas. $O$ segundo caso, aos 41 anos, apresentava além do quadro parkinsoniano, reflexo mandibular hiperativo, mas, também, arreflexia tendinosa global nos 4 membros, sem as alteraçōes sensitivas presentes no seu irmão. Havia tambêm sinais de degeneração retiniana no eletrorretinograma. Da mesma maneira, demonstrou dramática resposta a levodopa, porém logo desenvolvendo movimentos corêicos. $O$ pai dos pacientes, aos 73 anos iniciou tremor de repouso sem outras manifestaçðes parkinsonianas e faleceu aos 81 anos, devido a acidente vascular cerebral. Uma sobrinha dos dois pacientes apresentava pés cavos, sem anormalidades ao exame neurológico. Tratava-se, então, de dois casos de parkinsonismo juvenil (ou de início precoce, dependendo de como usemos as classificaçóes propostas) familiar, com sinais de degeneração de múltiplos sistemas. Não havia descrições semelhantes na literatura. O mecanismo de transmissāo hereditária aparentemente era autossômico dominante, com expressão variável. Os casos nāo diferiam muito de outros casos apresentados de parkinsonismo juvenil ou DP de início precoce, a não ser pelas manifestaçōes neurológicas de outros sistemas. Apresentaram a descrição patológica do primeiro caso: gliose na 6" camada do córtex; desmielinização da corona radiata; perda neuronal no globo pálido; gliose no núcleo subtalâmico; perda de neurônios no núcleo centromediano do tálamo e gliose no núcleo ventrolateral; na substância negra havia perda neuronal predominante na pars reticulata ( $40 \%$ de perda em relação aos normais), sem corpúsculos de Lewy ou degeneração neurofibrilar; havia também atrofia do tegmento da ponte; hipertrofia e vacuolização nas células da parte ventral da oliva bulbar; degeneração das colunas dorsais e gliose nos núcleos 
das colunas dorsais no bulbo; o núcleo denteado do cerebelo tinha pequena atrofia; a medula espinal era muito atrófica, com marcada degeneração da coluna dorsal; as raizes dorsais mostravam intensa desmielinizaçăo, menor nas raízes ventrais. Os autores concluiram por uma heredodegeneração, relacionada com o parkinsonismo juvenil, a atrofia dentato-rubro-palido-luisiana e a degeneração espino-cerebelo-nigral sendo as mais prováveis.

Um caso curioso de parkinsonismo de início precoce num paciente do sexo masculino, iniciado aos 24 anos, que evoluiu com quadro demencial a partir dos 30 anos, foi publicado em 1992, por Yoshimura et al. ${ }^{71}$. Seus pais eram consanguíneos. Aos 34 anos sintomas autonômicos eram proeminentes, tinha episódios frequentes de diarréia e perdia peso progressivamente. Em seus exames laboratoriais, a não ser por pequena elevação da fosfatase alcalina sérica e da desidrogenase láctica, nada fó encontrado. Faleceu aos 38 anos, após pneumonias de repetiçāo, num quadro de extrema demência e mutismo acinético. Os dados neuropatológicos, que já haviam sido publicados em outro trabalho, mostraram a presença de corpúsculos de Lewy difusamente em todo o córtex cerebral, ao lado das anormalidades habitualmente encontradas nos casos de DP clássica. $\mathrm{O}$ exame patológico das vísceras revelou aumento do pâncreas por maciça substituiçăo do tecido pancreático por gordura (pseudohipertrofia lipomatosa), com atrofia dos elementos glandulares exócrinos e preservação das ilhotas de Langerhans. O fígado apresentava histologia grosseiramente semelhante à da hepatite alcoólica, associada à presença difusa de corpos de Mallory ("Mallory bodies"), que possivelmente indicavam um distúrbio do metabolismo protêico. Concluiram sugerindo que distúrbio do metabolismo protêico comum aos três processos, desencadeado por um agente tóxico específico, endógeno ou exógeno poderia estar subjacente ao processo, causando os corpúsculos de Lewy difusos no cérebro, os corpos de Mallory no fígado e a degeneração lipomatosa do pâncreas.

Dwork et al. ${ }^{16}$ relataram uma família de pacientes parkinsonianos com herança autossômica dominante. que em três geraçðes apresentou 14 pacientes com parkinsonismo cujo início se deu entre a primeira e a quarta décadas da vida. Distonia era um fato proeminente em vários dos pacientes com início mais jovem. O paciente de início mais jovem foi aos dois anos e o mais tardio, aos 39 anos. O paciente que foi descrito em detalhe, era do sexo masculino, que aos 28 anos desenvolveu quadro de rigidez plástica generalizada, com tremor de repouso e bradicinesia. Doses moderadas de levodopa e tri-hexifenidil controlaram bem seus sintomas por vários anos, a partir do que apareceram discinesias e flutuaçōes "on-off". Aos 38, já não andava sem ajuda. Aos 44, ficava imobilizado a maior parte do dia. Foi submetido a uma cirurgia de implante de tecido autólogo da medula supramenal, que produziu bom efeito por vários meses. Após 8 meses, desenvolveu piora progressiva, com inatençăo e comportamento inadequado. Uma tomografia computadorizada cerebral revelou lesðes hiperdensas no lobo frontal direito e tálamo esquerdo, que à biópsia mostraram ser focos de glioblastoma multiforme. Faleceu um ano após. Os autores descreveram o exame neuropatológico postmortem: havia três focos de glioblastoma multiforme, nenhum deles em conexão direta com os locais dos implantes; a substância negra estava muito pálida; havia alteraçð̃es devidas ao edema, às hemiaçð̄es, consequências das mesmas e os detalhes dos implantes, os quais não iremos apresentar. No tocante ao parkinsonismo, as anormalidades se limitavam à substância negra, com grande perda neuronal na pars compacta e pars reticulata, com pouca gliose na pars compacta. Os neurônios remanescentes eram pouco pigmentados. Não foram encontrados corpúsculos de inclusăo de Lewy. O corpo estriado, globo pálido, núcleo olivar inferior, núcleo denteado, as vias palidofugais e o córtex cerebelar nāo revelavam anormalidades. Nos implantes não se encontraram células da medula adrenal vivas. Os autores fazem considerações sobre os achados neuropatologicos de relatos prévios, todos os que também já relatamos, nāo encontrando nenhum superponível ao do presente caso. Acharam que se aproximava mais do caso descrito por Yokochi et al. ${ }^{\boldsymbol{}}$, porém com perda nigral mais disseminada, não seletiva, mas o paciente nāo iniciou o quadro em idade tão baixa quanto o dos autores japoneses, nem apresentou fenômenos distônicos. Entretanto, outros membros da família apresentada por Dwork et al. ${ }^{16}$ apresentavam quadros semelhantes aos de Yokochi et al., com início muito precoce e distonia. Talvez a neuropatologia nestes outros casos pudesse ser mais próxima da descrita por Yokochi et al. ${ }^{20}$.

Um aspecto final a que devemos nos reportar é o relacionado com as evidências de que fatores arnbientais diversos podem ter relaçăo com a patogênese da DP, especialmente nos casos de pacientes mais jovens. A descoberta, no início da década passada de que o MPTP (1-metil-4-fenil-1,2,3,6-tetra-hidropiridina), um análogo de meperidina, obtido como subproduto na síntese desta substância, por jovens viciados em drogas na California, era capaz de produzir lesões cerebrais semelhantes às da DP, com sintomas clínicos de tipo parkinsoniano ${ }^{5,37,38}$, trouxe a atenção para o papel que os fatores ambientais, como contaminantes químicos, industriais, toxinas exógenas e produtos defensivos agrícolas, entre outros, podem desempenhar na gênese da DP. Logo a seguir, o MPTP transformou-se na principal ferramenta de pesquisa no campo da origem da DP, servindo como o veículo de obtenção de modelos animais de DP, utilizados em pesquisas clínicas e famacológicas ${ }^{32,36,39}$. O grupo canadense 
liderado por Barbeau, produziu vários trabalhos na linha dos fatores ambientais, assim como na da ecogenética da DP, produzindo as primeiras evidências de que os pacientes que habitavam zonas rurais (onde o consumo de agrotóxicos era maior), em zonas nas cercanias de complexos industriais, especialmente de indústrias químicas, siderúrgicas e de papel, eram estatisticamente mais propensos a desenvolver a DP. Uma susceptibilidade genética poderia estar envolvida também em certos indivíduos, tomando-os propensos a desenvolver a DP, quando em longo contacto com determinadas substâncias do ambiente, naturais ou não. Este grupo sugeriu que uma deficiência do citocromo hepático P450 (anomnalidade com herança recessiva), envolvido nos processos de detoxificação, que é demonstrada por um comprometimento da hidroxilação de debrisoquina, era mais comum nos pacientes de DP com início abaixo dos 40 anos ${ }^{6-10}$. Outras evidências de que pacientes com início precoce de DP estāo mais sujeitos a influências de fatores ambientais na gênese da doença, também haviam sido observadas pelo grupo de Rajput ${ }^{\text {sa-s2 }}$, da província de Saskatchewan no Canadá, desde 1984. Demonstraram correlação entre a exposiçāo numa idade muito precoce (infância e adolescência) ao ambiente de vida rural e com o consumo habitual de água de poço, com o desenvolvimento posterior de DP idiopática, especialmente quando o início dos sintomas se dava abaixo dos 40 anos. Entretanto não foram capazes de apontar quais elementos químicos existentes na água de poço da região poderiam estar relacionados com a DP. Estes mesmos fatores ambientais foram também estudados pelo grupo de Tanner, na área de Chicago, com estudos epidemiol6gicos com pacientes parkinsonianos jovens, chegando a resultados semelhantes ${ }^{60-62}$. Entretanto, uma pesquisa bem controlada, comparando grupo de pacientes jovens, com início dos sintomas abaixo dos $\mathbf{4 0}$ anos, com grupo de pacientes com início acima dos 60 anos, ambos confrontados com grupo controle, no sentido de se buscar evidências epidemiológicas de fatores ambientais, como os já citados, não conseguiu demonstrar qualquer ligação tanto nos casos jovens, como nos mais idosos, com qualquer fator ambientals9. Em nosso meio, o grupo liderado por nós, motivado por dois pacientes com parkinsonismo de início precoce, investigou a possibilidade de uma contaminação a longo prazo com produto orgânico de manganês (Maneb), utilizado como fungicida em cultura de tomates, ser o responsável pela gênese da enfermidade. $O$ grupo demonstrou sinais sugestivos de contaminação crônica mangânica, com sinais parkinsonianos frustros em uma população de indivíduos que manipulavam o produto ${ }^{17}$. Extensas revisões sobre a influência dos fatores ambientais na gênese da DP podem ser consultadas ${ }^{128,29,62}$, assim como sobre o tema de parkinsonismo de início precoce em geral ${ }^{20,21,28}$. Em 1994, estudamos todos os nossos pacientes com doença de Parkinson cujo início dos sintomas se dera até os 40 anos de idade. Entre 1278 pacientes catalogados com tendo parkinsonismo (não apenas doença de Parkinson) do ambulatório de enfermidades extrapiramidais da Neurologia da Escola Paulista de Medicina e da nossa clínica privada, pudemos identificar e estudar 58 pacientes com aquelas características ${ }^{2}$. Atualmente este número já atinge 67 pacientes. Estudamos diversas características clínicas e epidemiológicas e os resultados serão publicados separadamente.

De todo o exposto até o momento, pode-se depreender que uma série de pontos ainda estão muito controversos em relaçāo aos pacientes que desenvolvem DP ou parkinsonismo em idades muito jovens, abaixo do esperado. O primeiro ponto seria a respeito da delimitação da idade abaixo da qual já se poderia considerar como precoce o início. Quarenta anos seria a idade de corte ideal ou abaixo dos 30 , ou mesmo dos 20 anos seria mais adequado? Um outro, relacionável com a denominaçăo que se deva usar para definir os pacientes. Aqui, nāo vale apenas o aspecto etário, temporal do início dos eventos, isto é, se vamos denominá-los de casos juvenis, jovens, de início precoce ("juvenile, early-onset, young-onset"), mas também, e talvez mais importante ainda, se se vai atribuir-lhes a denominação específica de doença de Parkinson juvenil ou de início precoce, ou mais genérica, de parkinsonismo juvenil ou de início precoce. Nestes aspectos, entram as discussões acerca da patologia básica. São casos de doença de Parkinson clássica, apenas que se iniciando em uma idade mais baixa? Sāo casos representativos de outra patologia básica, apenas semelhante à da DP? Serăo processos patológicos intrinsecamente diferentes que culminam na produçāo de um efeito final semelhante? Os casos com antecedentes familiares nos variados graus (irmāo em primeiro grau, outro parente em primeiro grau, segundo grau, etc) exprimindo influência gênica, serão a expressão de uma patologia diversa da que ocorre nos casos sem antecedentes familiares positivos? $O$ pacientes abaixo dos 20 anos devem ser entendidos como pertencendo a um subgrupo especial, provavelmente sendo a consequência de outras enfermidades? A curiosa associação de distonia, especialmente a que inaugura o quadro clínico, em idades muito baixas, com localização nas pernas e pés, exprime uma forma transicional entre a distonia-dopa-responsiva, a doença de Parkinson precoce $e$ as formas com sintomas clínicos flutuantes durante o dia? É possível que este enorme grupo de pacientes que apresenta algumas peculiaridades comuns, como a sindrome parkinsoniana precoce, a presença de fenômenos distônicos, a alta prevalência de antecedentes familiares, a resposta acentuadamente pronta a doses reduzidas de levodopa, com alta possibilidade de desenvolvimento de discinesias e flutuaçöes do rendimento terapêutico 
a curto prazo, seja uma coleçāo de entidades clínicas diferentes, compartilhando mecanismos patogênicos semelhantes. Ou então, o cérebro jovem estaria apresentando uma maneira diferente de reagir a uma agressão comum. E mais, indivíduos com susceptibilidades geneticamente conferidas, em idades precoces, poderiam estar sendo agredidos por fatores ambientais ainda não definidos e exprimindo-se de maneira ora semelhante, ora diversa.

Com tais questões ainda em aberto, e levando-se em consideração o número relativamente pequeno de pacientes apresentados em cada série, e, ainda, a diversidade de aspectos clínicos envolvida numa parcela considerável de casos, torna-se imperativo que todos os estudiosos do assunto, aqueles que detêm parcr.!as significativas de pacientes sob controle, voltem-se a seus casos, estudando-os sob forma sistemática e apresertem seus resultados à comunidade, de modo a propiciar com isto uma melhor possibilidade de compreensão e esclarecimento.

\section{REFERÊNCIAS}

1. Andrade LAF. The role of the environmental factors in the genesis of Parkinson's disease. In Caraceni T, Nappi G, (eds) Focus on Parkinson's disease Milano: Masson, 1991:67-78.

2. Andrade LAF. Contribuição ao estudo da doença de Parkinson de inf́cio precoce: análise clínica de 58 pacientes. Tese de Livre-Docência. Escola Paulista de Medicina (Universidade Federal de São Paulo).São Paulo, 1994.

3. Araújo AQC, Miranda SBM. Doença de Segawa: distonia progressiva sensivel à L-Dopa: relato de um caso. Arq Neuropsiquiatr 1993;51:532- 536.

4. Askenazy JJM, Mendelson L, Keren O, Braun Z. Juvenile Parkinson's disease and its response to L-dopa therapy. J Neural Transm 1990;2:23-30.

5. Ballard PA. Tetrud JW, Langston JW. Permanent human parkinsonism due to 1-methyl-4-phenyl-1,2,3,6-tetrahydropyridine (MPTP):seven cases. Neurology 1985;35:949-56.

6. Barbeau A, Cloutier RT, Plasse L, Paris S. Environmental and genetic factors in the etiology of Parkinson's disease. In Yahr MD, Bergmann KJ (eds) Adv Neurol, Vol. 45. New York: Raven Press 1986;299-306.

7. Barbeau A, Pourcher E. New data on the genetics of Parkinson's disease. Can J Neurol Sci 1982;9:53-66.

8. Barbeau A. Roy M. Genetic susceptibility, environmental factors and Parkinson's disease. Proceedings of the VIII International Symposium on Parkinson's Disease, New York: June, 9-12, 1985:13.

9. Barbeau A, Roy M, Bernier G, Campanella G, Paris S. Ecogenetics of Parkinson's disease: prevalence and environmental aspects in rural areas. Can J Neurol Sci 1987;14:36-41.

10. Barbeau A, Roy M, Paris S, Cloutier T, Plasse L, Poirier J. Ecogenetics of Parkinson's disease: 4-hydroxylacion of debrisoquine. Lancet 1985:2:1213-6.

11. Bury JS. Two cases of paralysis agitans in the same family in which mprovement followed the administration of hyoscine. Lancet 1902;1: 1097.

12. Butterfield PG, Valanis BG, Spencer PS, Lindeman CA, Nutt JG. Environmental antecedents of young-onset Parkinson's disease. Neurology 1993;43:1150-1158.

13. Carlier G, Dubru JM. Familial juvenile parkinsonism. Acta Paediat Belg 1979;32:123-127.

14. Clough CG. Mendoza M. Yahr MD. A case of sporadic juvenile Parkinson's disease. Arch Neurol,1981;38:730-731.

15. Davison, D. Pallido-pyramidal disease. J Neuropath Exp Neurol,1947;13:50-59.

16. Dwork AJ, Balmaceda C, Fazzini EA, Mac Collin M, Côté LA, Fahn S. Dominantly inherited, early-onset parkinsonism: neuropathology of a new form. Neurology 1993;43:69-74.

17. Ferraz HB, Bertolucci PHF, Pereira JS. Lima JGC, Andrade LAF. Chronic exposure to the fungicide maneb may produce symptoms and signs of CNS manganese intoxication. Neurology 1988;38:550-553.

18. Ferreiro JL, Pugliese MI, Caride AE. Parkinsonismo juvenil: a proposito de 18 casos. Medicina (Buenos Aires) 1991;51:204-208.

19. Furukawa $Y$, Nishi K, Kondo T, Mizuno Y,Narabayashi H. Juvenile parkinsonism: ventricular CSF biopterin levels and clinical features. J Neurol Sci, 1992;108:207-213.

20. Gershanik OS. Parkinsonism of early onset. In Jankovic J. Tolosa E (eds). Parkinson's disease and movement disorders, Baltimore: Urban \& Schwarzenberg, 1988:191-204.

21. Gershanik OS. Parkinsonism of early onset. In Jankovic J, Tolosa E (eds) Parkinson's disease and movement disorders. Baltimore: Williams \& Wilkins, 1993:235-252.

22. Gershanik OS, Leist A. Juvenile onset Parkinson's disease. In Yahr MD, Bergmann KJ. Adv Neurol Vol. 45. New York: Raven Press 1986:213-216.

23. Gershanik OS, Nygaard TG. Parkinson's disease beginning before age 40. In Streifler MB, Korczyn AD, Melamed E, Youdim MBH (eds). Adv Neurol Vol 53 New York: Raven Press, 1990:251-258.

24. Gibb WRG, Lees AJ. A comparison of clinical and pathological features of young- and old-onset Parkinson's disease. Neurology, 1988;38:1402-1406.

25. Gibb W, Narabayashi H, Yokochi M, lizuka R. Additional pathological observations in juvenile onset parkinsonism with dystonia (Abstr.). Neurology 1989;39 (Suppl 1.): 139.

26. Gibb WRG, Narabayashi H, Yokochi M, Iizuka R, Lees AJ. New pathologic observations in juvenile onset parkinsonism with dystonia. Neurology 1991;41:820-822.

27. Giovannini P, Piccolo S, Genitrini P, Soliveri F, Girotti G, Geminiani G, Scigliano G, Caraceni T. Early-onset Parkinson's disease. Mov Disord 1991;6:36-42. 
28. Golbe L.I. Young-onset Parkinson's disease: a clinical review. Neurology 1991;41:168-173.

29. Golbe L.I. Risk factors in young-onset Parkinson's disease. Neurology 1993;43:1641-1643.

30. Hoehn MM. Age distribution of patients with parkinsonism. J Am Geriat Soc 1976;24:79-85.

31. Hunt J.R. Progressive atrophy of the globus pallidus. Brain 1917;40:58-148.

32. Jenner $P$, Marsden CD. MPTP-induced parkinsonism in primates and its use in the assessment of novel strategies for the treatment of Parkinson's disease. In Rose FC (ed). Current problems in neurology (6): Parkinson's disease:clinical and experimental advances. London: John Libbey, 1988:149-162.

33. KollerW, O'Hara R, Weiner W, Lang A, Nutt J, Agid I, Bonnet A.M, Jankovic J. Relationship of aging to Parkinson's disease. In Yahr MD, Bergmann KJ (eds). Adv Neurol, Vol.45.New York: Raven Press, 1986:317-321.

34. Kostic V, Przedborski S, Flaster MS, Sternic N. Early development of levodopa-induced dyskinesias and response fluctuations in young-onset Parkinson's disease. Neurology 1991;41:202-205.

35. Krabbe KH. Nogle Undersogelser over Paralysis Agitans. Bibliotek for Laeger, 1923;115:355-363.

36. Langston J.W. MPTP-induced parkinsonism: how good a model is it? In Fahn S (ed). Recent developments in Parkinson's disease. New York: Raven Press, 1986:119-126.

37. Langston JW, Ballard PA. Parkinson's disease in a chemist working with l-methyl-4-phenyl-1,2,3,6-tetrahydropyridine (MPTP). N Engl J Med 1983;309:310.

38. Langston JW, Ballard P, Tetrud JW, Irwin I. Chronic parkinsonism in humans due to a product of meperidine analog synthesis. Science 1983;219:979-980.

39. Langston JW, Langston EB, Irwin I. MPTP-induced parkinsonism in human and non-human primates; clinical and experimental aspects. Acta Neurol Scand 1984;70: 49-54.

40. Lima AB, Levy A, Castro Caldas A, Neves G, Lopes G, Sampaio C. Parkinson's disease before age 30. In Narabayashi H, Nagatsu T, Yanagizawa N, Mizuno Y. Adv Neurol, Vol 60. New York: Raven Press, 1993:553-557.

41. Lima B, Neves G, Nora M. Juvenile parkinsonism: clinical and metabolic characteristics. Proceedings of the VIII International Symposium on Parkinson's Disease, New York: June 9-12,1985 P6.

42. Lima B, Neves G, Nora M. Juvenile parkinsonism: clinical and metabolic characteristics. J Neurol Neurosurg Psychiatry 1987;50:345-348.

43. Martin WE, Resch JA, Baker AB. Juvenile parkinsonism. Arch Neurol 1971;25:494-500.

44. Mayer JM, Mikol J, Haguenau M, Dellanave J, Pépin B. Familial juvenile parkinsonism with multiple systems degenerations: a clinicopathological study. J Neurol Sci 1986;72:91-101.

45. Mjones H. Paralysis agitans: a clinical and genetic study. Acta Psychiatr Neurol 1949;54(Suppl):1-195.

46. Nygaard TG, Marsden CD, Duvoisin, RC. Dopa-responsive dystonja. In Fahn S, Marsden CD, Calne DB (eds) Adv Neurol, Vol 50. New York: Raven Press, 1988:377-384.

47. Ota Y, Miyoshi S, Ueda O, Mukai T, Maeda A. Familial paralysis agitans juvenilis: clinical, anatomical and genetic study. Folia Psychiat Neurol Japonica 1958;12:112-121.

48. Parkinson J. An essay on the shaking palsy. Sherwood, Neely \& Jones, 1817. Ed. fac-simile in Med Class 1938;3:964-997.

49. Quinn N, Critchley P, Marsden CD. Young onset Parkinson's disease. Mov Disord, 1987;2:73-91.

50. Rajput AH, Stern W, Christ A, Laverty W. Etiology of Parkinson's disease: environmental factor(s). Neurology 1984;34:(Suppl)207.

51. Rajput AH, Uitti RJ, Stern W, Laverty W. Early onset Parkinson's disease and childhood environment. In Yahr MD, Bergmann KJ. Adv Neurol, Vol 45. New York: Raven Press, 1986:295-297.

52. Rajput AH, Uitti RJ, Stern W, Laverty W, O'Donell K, Yuen WK, Dua A. Geography, drinking water chemistry, pesticides and herbicides and the etiology of Parkinson's disease. Can J Neurol Sci 1987;14:414-418.

53. Sachdev KK, Singh N, Krishnamoorty MS. Juvenile parkinsonism treated with levodopa. Arch Neurol, 1977;34:244-245.

54. Sardinha LA, Braga AA, Quagliato EMAB. Parkinsonismo juvenil familiar: apresentaçāo de 3 casos e revi são da literatura. Arq Neuropsiquiatr 1988; 46(Supl):223.

55. Scaff M, Barbosa ER, Assis JL, Canelas HM. Parkinsonismo juvenil: consideraçŏes a respeito de 10 casos. Arq Neuropsiquiatr, 1980;38:385- 390.

56. Scott RM, Brody JA. Benign early onset Parkinson's disease: a syndrome distinct from classic postencephalitic parkinsonism. Neurology 1971;21:366-368.

57. Segawa M, Hosaka A, Miyagawa F, Nomura Y, Imai H. Hereditary progressive dystonia with marked diurnal fluctuation. In Eldridge R, Fahn S.Adv.Neurol, Vol.14. New York: Raven Press, 1976:215-233.

58. Segawa M, Nomura Y, Kase M. Hereditary progressive dystonia with marked diumal fluctuation: clinico-pathophysiological identification in reference to juvenile Parkinson's disease. Proceedings of the VIII International Symposium on Parkinson's Disease, New York: Raven Press 1985:61.

59. Stern M, Dulaney E, Gruber SB, Golbe L, Bergen M, Hurtig H, Gollomp S, Stolley P. The epidemiology of Parkinson's disease: a case-control study of young-onset and old-onset patients. Arch Neurol 1991;48:903-907.

60. Tanner CM, Chen B, Wang WZ, Peng ML, Liu ZL, Liang XL, Kao LC, Gilley DW. Schoenberg, B.S. Environmental factors in the etiology of Parkinson's disease. Can J Neurol Sci 1987;14:419-423.

61. Tanner CM, Grabler P, Goetz CG. Occupation and the risk of Parkinson's disease: a case-control study in young-onset patients. Neurology 1990; 40 (Suppl 1):422.

62. Tanner CM, Langston JW. Do environmental toxins cause Parkinson's disease? A critical review. Neurology 1990; 40 (Suppl 3):17-30.

63. Teravainen H, Forgath L, Hietanen M, Schulzer M, Schoenberg B, Calne D.B. The age on onset of Parkinson's disease: etiological implications. Can J Neurol Sci, 1986;13:317-319.

64. Van Bogaert L. Contribution clinique et anatomique a l'étude de la paralysie agitante juvenile primitive. Rev Neurol (Paris) 1930;11:315-326.

65. Willige H. Uber Paralysis Agitans in Jugendlichem Alter. Z Gesamte Neurol Psychiatr 1911;4:520-587. 
66. Yamamura Y, Sobue I, Ando K, lida M, Yanagi T, Kono C. Paralysis agitans of early onset with marked fluctuation of symptoms. Neurology 1973;23:239-244.

67. Yokochi M. Juvenile Parkinson's disease: 1. Clinical aspects. Adv Neurol Sci (Tokyo) 1979;23:1048-1059.

68. Yokochi M. Juvenile Parkinson's disease: Pharmacokinetic study. Adv Neurol Sci (Tokyo) 1979;23:1060-1073.

69. Yokochi M, Narabayashi H. Clinical characteristics of juvenile Parkinsonism. In Rose FCR, Capildeo R. Research progress in Parkinson's disease. Tunbridge Wells, Kent, R. Pitman Medical, 1981:35-39.

70. Yokochi M, Narabayashi H, Lizuka R, Nagatsu T. Juvenile parkinsonism some clinical, pharmacological, and neuropathological aspects. In Hassler RG, Christ JF. Adv Neurol, Vol 40. New York: Raven Press, 1984;407-413.

71. Yoshimura N, Hayashi S, Fukushima Y. Diffuse Mallory bodies in the liver, diffuse Lewy bodies in the brain and diffuse fat replacement (lipomatous pseudohypertrophy) of the pancreas in a patient with juvenile Parkinson's disease. Acta Pathol Japonica 1992;42:826-831. 